\title{
Determination of the elastic properties of woven composite panels for Lamb wave studies
}

\author{
W.H. Ong ${ }^{\mathrm{a}^{*}}$, N. Rajic ${ }^{\mathrm{b}}$, W.K. Chiu ${ }^{\mathrm{a}}$, C. Rosalie \\ ${ }^{a}$ Department of mechanical and aerospace engineering, Monash University, Wellington Rd, Clayton, \\ VIC 3800, Australia \\ ${ }^{\mathrm{b}}$ Defence Science \& Technology Group (DSTO), 506 Lorimer St, Fishermans Bend, VIC 3207, \\ Australia \\ * Corresponding author: wern.ong@ monash.edu
}

\begin{abstract}
Typically, numerical simulations of Lamb wave propagation are done using material properties which originate from tensile testing. This approach is well established in relation to isotropic homogenous structures such as aluminium plates. However if this approach is used for woven composites such as carbon fibre reinforced plastics (CFRP), inaccuracies can arise that stem from vastly different stress distributions, strain rates and amplitudes during Lamb wave propagation. In order to account for this, an approach is presented where the elastic properties in a numerical Lamb wave model are optimised to achieve good correlation between model predictions and experimental observations. Since the material properties are determined under a Lamb wave propagation regime, the strain rates and amplitudes are consistent with the intended modelling application. The approach is validated with an experimental case study involving a M18/G939 carbon-epoxy system. The methodology is shown to yield property estimates that furnish simulations that closely match observed behaviours. The optimised properties were significantly different to those supplied by the manufacturer, as much as $52 \%$ for the in-plane stiffness. The findings demonstrate that large errors are possible if elastic properties determined using conventional quasi-static testing are used in Lamb wave simulations pertaining to woven composite materials.
\end{abstract}

Keywords: woven composite; CFRP plate; lamb wave simulation; elastic properties

\section{Introduction}

Composite materials such as woven carbon fibre reinforced plastics (CFRP) have become increasingly prevalent in aerospace applications. Lamb wave based methodologies are an important class of monitoring technique that can be used to monitor defects in these structures. In determining the efficacy of Lamb wave based methodologies, numerical simulations are relied upon for predictions and insight. The majority of studies in the literature address isotropic materials such as aluminium [1-6]. When modelling the propagation of Lamb waves in such media, the widely practiced approach is to rely on elastic properties obtained from published material data. In most cases such a result yields acceptable levels of simulation accuracy. For example, Ong et al [7] presented a set of dispersion curves derived from measured multi-modal Lamb waves in an aluminium test plate over a frequency bandwidth between $200 \mathrm{kHz}$ and $1000 \mathrm{kHz}$. They reported excellent agreement between the experimental results and theoretical predictions from DISPERSE [8]. 
The elastic constants used for the aluminium plates were selected from the DISPERSE material library which is similar to that available in finite element software.

The literature shows that using published material properties can be a valid approach when applied to composite panels made from unidirectional laminates. $\mathrm{Ng} \mathrm{C}-\mathrm{T}$ [9] presented a set of results on the numerical modelling of Lamb waves in composite plates made from unidirectional material. Adequate agreement with their experimental data was achieved by using engineering constants from constituent fibre and resin materials to evaluate the equivalent material properties using micromechanics theory. Similarly, Li et al [10] also achieved good agreement with the use of material properties determined using mechanical tests in their dynamic Lamb wave simulations.

To date, there is limited work reporting on the Lamb wave dispersion characteristics of composite plates made from woven CFRP. An accurate set of elastic properties of woven composite plate is essential for an adequate theoretical prediction of the Lamb wave dispersion characteristics in this material. Unlike composite plates made from unidirectional fibres, woven composites are characterised by the weave parameters. Naik [11] reported that the stiffness of woven composite panels is governed by weave parameters such as weave architecture, yarn sizes, yarn spacing and yarn crimp. The concept of the repeating unit cell (RUC) was used to help define the elastic properties of woven composites [12]. Gommer et al [13] applied similar RUC concept to determine the elastic properties of knitted fabric reinforced composites. The inherent difficulties in determining the elastic properties of woven material are highlighted in these works. Gommer et al [13] showed large discrepancies between the experimentally obtained and predicted values of Young's modulus in a woven composite when using Voigt and Reuss methods[14].

It is known that the Lamb wave modes supported by a test plate can have length-scales that are comparable to the RUC of a woven composite plate [15]. Poe et al [16] reported a strain gauge length less than the RUC length can return a modulus up to $17 \%$ different to that measured by extensometer or longer gauge lengths. This suggests that woven composites have small scale stiffness inhomogeneities; an observation that is supported by the literature [17-19]. Mishra [19] illustrates this with finite element modelling showing substantial stress and strain variations within a RUC under simple tensile loading.

Malcolm et al [20] recognised that although the fibre waviness in woven composites can reduce the modulus under initial loading, the straightening of the wavy fibre tow will lead to an increase in the modulus with increasing tensile loading. In compression, the modulus of a woven composite is expected to be lower than a laminated unidirectional fibre composite due to fibre waviness. This infers that the elastic modulus at very low loads will be dependent on the applied force supported by the material. Given that the strain levels associated with Lamb waves are small, it can be inferred that using the elastic modulus of a woven composite plate determined from quasi-static tensile testing for the simulation of Lamb wave propagation may lead to significant errors (see Ong et al [15]). For predictive Lamb wave modelling, a more reasonable approach is to determine the elastic constants using a loading regime that more closely matches the strain rates and strain amplitudes of Lamb wave propagation.

This paper reports on the development of a method to determine the elastic properties in a woven CFRP composite plate. The proposed method employs a numerical model and particle swarm optimisation (PSO) [21]. The PSO is a stochastic optimisation technique based on the movement of swarms. Prior to this study, a verification of the PSO was performed on aluminium. The results from that study are described in Ong et al [7]. Optimisation of the material properties is achieved by 
comparing outputs from the numerical model against experimental data for a particular Lamb wave propagation regime. This ensures the optimised properties are relevant to Lamb wave simulation.

The paper begins with a brief explanation of some experimental details including the structure of the composite specimen and the method used to determine the dispersion curves for the first four modes. This data is used to illustrate the inaccuracies in the published material properties. A brief explanation of the PSO is presented along with descriptions of the finite element model and objective function. The optimised material properties are then applied to simulations that are compared to experimental observations.

\section{Specimen and measurement of properties}

The composite specimen was fabricated using Hexcel M18 prepreg with woven carbon G939 fabric. The plate to be characterised comprised 16 plies in the layup $[0 / 90,+/-45,+/-45,0 / 90,0 / 90,+/-45,+/-$ $45,0 / 90]_{\mathrm{s}}$. Such a sequence leads to a quasi-isotropic structure. After curing, the plate was trimmed to a planar dimension of $400 \mathrm{~mm}$ x $400 \mathrm{~mm}$. A $\varnothing 10 \mathrm{~mm} \times 1 \mathrm{~mm}$ thick Ferroperm piezoceramic disc of type Pz27 was placed at the centre of the plate as shown in Figure 1.

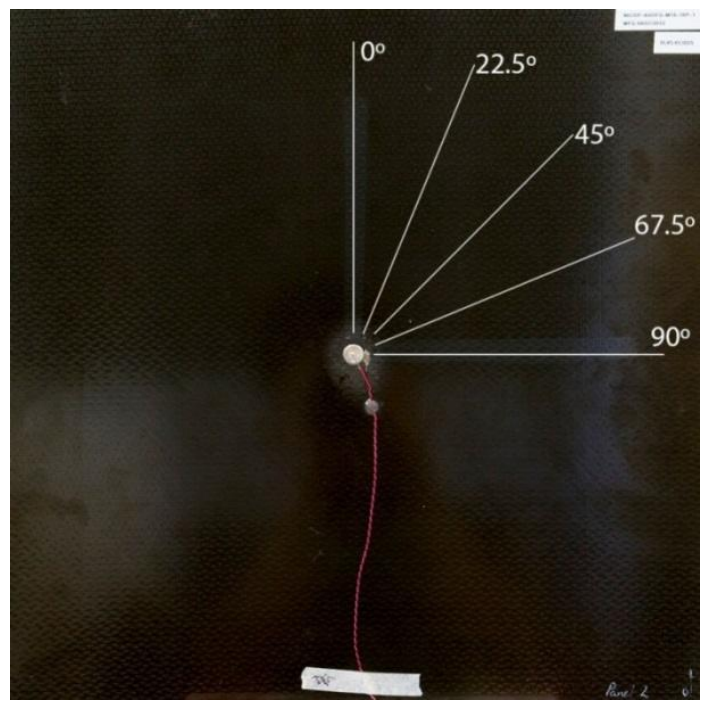

Figure 1 Photograph of specimen showing scan lines.

Hexcel [22] quotes a nominal ply thickness of $0.227 \mathrm{~mm}$, which implies a $3.63 \mathrm{~mm}$ final plate thickness for 16 plies. Measurements taken on the plate edges differed from this nominal value, revealing an uneven thickness. Additional measurements were taken across the plate using a dial indicator and precision surface block. The thickness of the plate was measured along the lines indicated in Figure 1. The thickness profiles for each line are shown in Figure 2. The plate centre was approximately $4.4 \mathrm{~mm}$ thick, while the thickness approached $3.9 \mathrm{~mm}$ at the edges. The thickness profile data was used to calculate the volume of the plate which was used in combination with a measurement of the plate mass to calculate the material density $\left(1434 \mathrm{~kg} / \mathrm{m}^{3}\right)$. Figure 3 defines the RUC of the material, which in the present case was approximately $7.6 \mathrm{~mm} \times 7.6 \mathrm{~mm}$. 


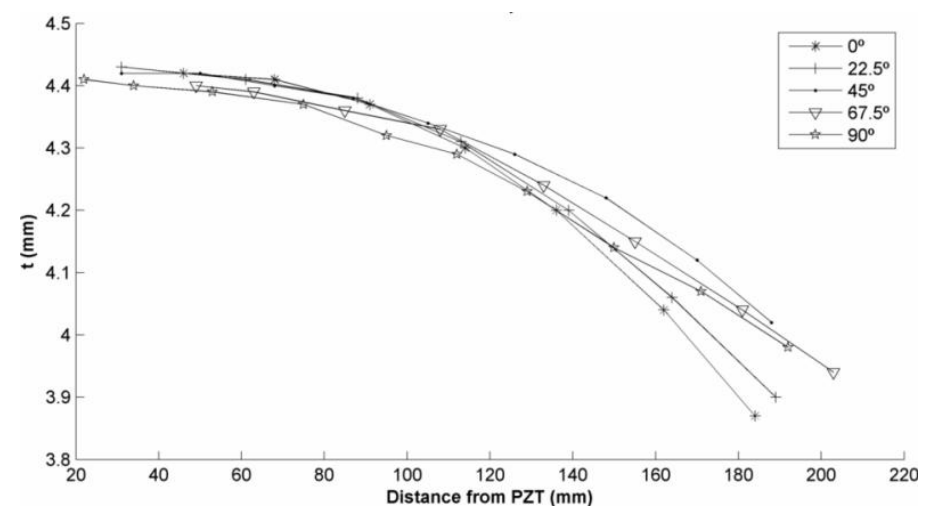

Figure 2 Thickness measured across lines indicated in Figure 1.

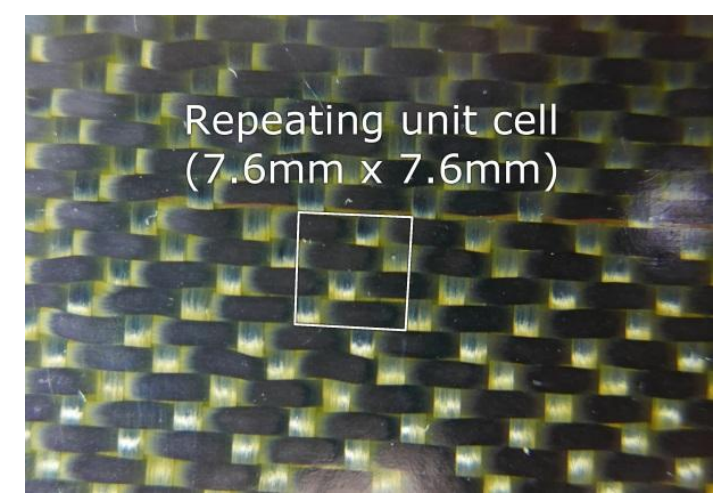

Figure 3 Photograph of CFRP test specimen showing 4 harness satin weave. Repeating unit cell is overlayed.

The initial properties are summarised in Table 1 and consist of the published in-plane modulus from the manufacturer and general woven CFRP properties for the remainder. Note $v_{23}$ is the "minor" Poisson's ratio and is related to the "major" Poisson's ratio $v_{13}$ by the relation shown in Equation 1 such that there are only 4 independent properties. These properties are used as the initial estimate for the optimisation problem described later in the paper. The coordinate system used in defining the properties is shown in Figure 4.

Table 1 Initial material properties.

\begin{tabular}{|c|c|}
\hline $\mathrm{E}_{11}=\mathrm{E}_{33}(\mathrm{GPa})$ & 67 \\
\hline $\mathrm{E}_{22}(\mathrm{GPa})$ & 8.6 \\
\hline $\mathrm{G}_{12}=\mathrm{G}_{13}=\mathrm{G}_{23}(\mathrm{GPa})$ & 5 \\
\hline$v_{12}=v_{13}$ & 0.35 \\
\hline$v_{23}$ & 0.045 \\
\hline
\end{tabular}

$$
v_{23}=E_{22} \frac{v_{12}}{E_{11}} \quad \text { Equation } 1
$$

\section{Laser vibrometry test facility}

The dispersion curves of the test plate were calculated from data acquired using a laser vibrometry (LV) facility described in detail in $[1,2]$. The piezoceramic disc bonded to the plate was made to actuate Lamb waves by applying a $50 \mathrm{~V}$ peak-peak drive signal from a Krohn-Hite 7602 amplifier. 
Out-of-plane displacements associated with the Lamb waves were detected on the plate surface by a Polytec OFV 505 laser vibrometer. The positioning of the laser vibrometer relative to the plate was controlled by a stepper-motor driven high resolution X-Y table, set to 5000 steps per mm.

To obtain the reference dispersion curves, the plate was scanned along the $0^{\circ}$ line shown in Figure 1 . The number of points scanned on this line resulted in a spatial resolution of $0.215 \mathrm{~mm}$ which is approximately a factor of ten shorter than the shortest wavelength studied. Multiple data sets were captured along this line using Hanning windowed sinusoids with centre frequencies ranging from 100 $\mathrm{kHz}$ to $500 \mathrm{kHz}$ in $50 \mathrm{kHz}$ intervals. Out-of-plane displacement was recorded on both sides of the plate as shown in Figure 4, which was achieved by turning the plate over on the laser vibrometry rig. Alignment of the two line scans was ensured by referencing against precise markings measured from the plate edge by Vernier calipers. An alignment error of $+/-0.1 \mathrm{~mm}$ is expected from this approach which is acceptable given the shortest wavelength is approximately $3 \mathrm{~mm}$. Figure 5 shows raw LV data from the top and bottom of the plate. The responses comprise contributions from both the fundamental anti-symmetric (A) and symmetric (S) modes. In theory for a given laser vibrometer scan head location, for instance when measuring $\mathrm{u}_{\mathrm{z} \text {,top }}$ (Figure 4), the $\mathrm{S}$ modes will displace the top surface towards and the bottom surface away from the scan head on a peak; conversely A modes will displace both the top and bottom surfaces towards the scan head on a positive peak. Therefore, subtraction or addition of the two data sets was used to separate the A/S modes as shown in Figure 5. This is especially important in the region of the dispersion curves where the $\mathrm{S}$ and $\mathrm{A}$ modes are in close proximity. The separation of these modes was done to facilitate the algorithmic implementation of the optimisation process as described shortly.

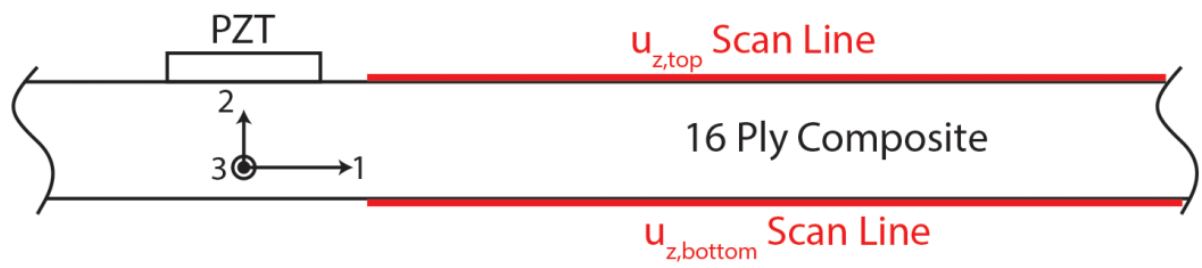

Figure 4 Schematic showing additional scan line and coordinate system.

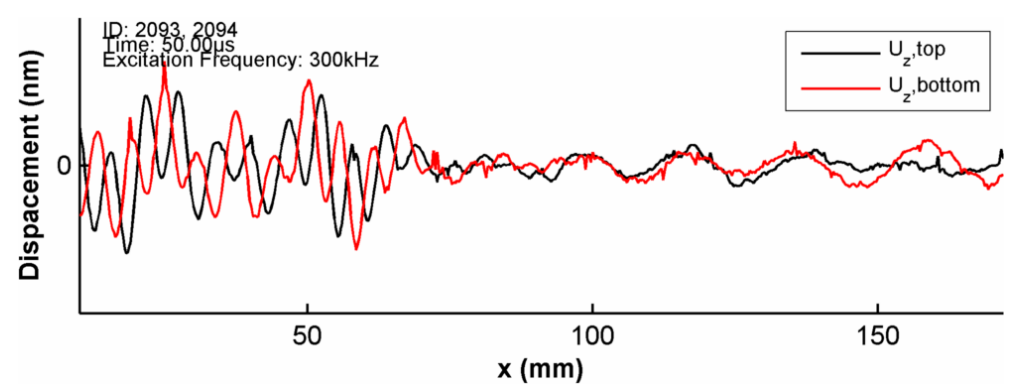

a)

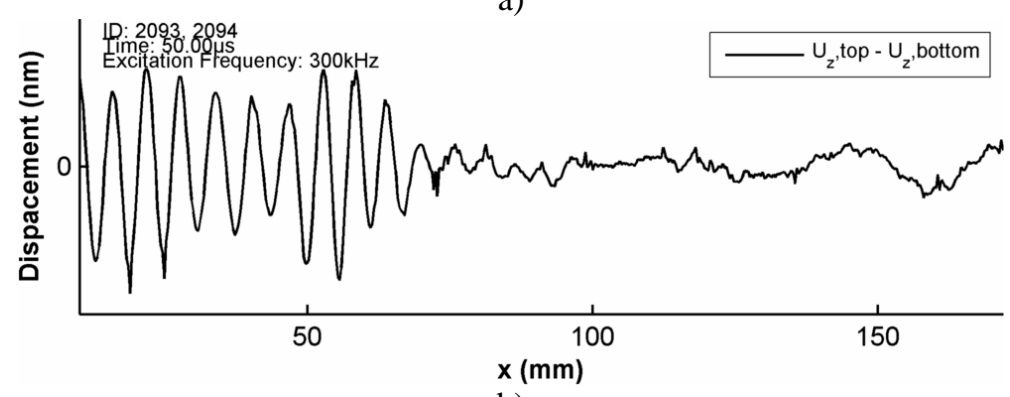

b) 


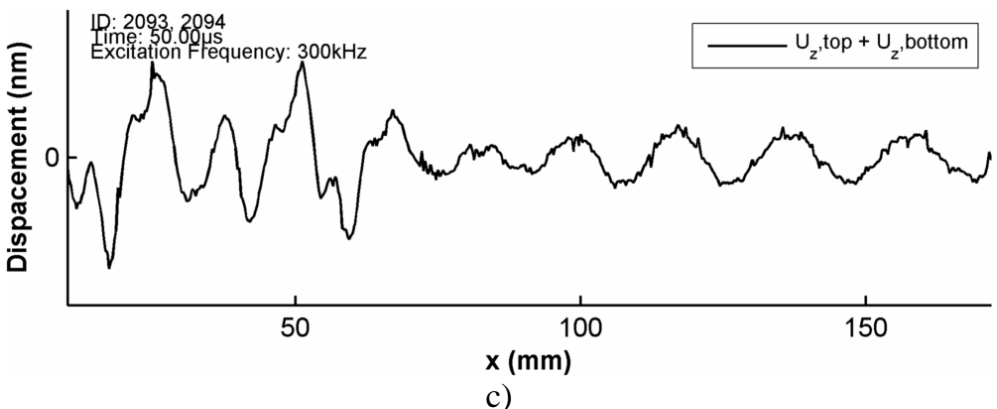

Figure 5 Displacement data at $50 \mu$ s with $300 \mathrm{kHz}$ excitation. a) Raw $\mathrm{LV}$ data from top and bottom scans. b) Antisymmetric mode contribution. c) Symmetric mode contribution.

\section{Material property determination using dispersion characteristics}

This section of the paper describes the formulation of the optimisation problem. In order to determine the dispersive characteristics, the displacement-time data captured by LV and described in the previous section was transformed using a 2D Fast Fourier Transform (FFT) [23]. A finite element (FE) model created in COMSOL provided the calculated dispersion curves. COMSOL was selected to model the specimen due its straightforward interface with MATLAB, which was the environment used to implement the PSO algorithm. Each ply in the laminate was modelled individually and mated together at their interfaces in a 2D plane-strain model. The plate model was created in the 1-2 plane using the coordinate system stated in Figure 4 and made $240 \mathrm{~mm}$ long such that the incident wave dominated the $150 \mu$ s time window used in the analysis. Each ply was assigned a thickness of 0.269 $\mathrm{mm}$ resulting in a total thickness of $4.3 \mathrm{~mm}$ which is consistent with an average of the plate thickness measurements described previously. The node spacing was set to $0.4 \mathrm{~mm}$ providing greater than 8 nodes per wavelength at all frequencies. The displacement-time data from this model was transformed by 2D FFT as was done for the experimental LV data.

As previously mentioned, in order to match the dispersive characteristics of the model to that extracted from experimental LV, a PSO algorithm was employed. The PSO was chosen for its capacity to handle non-linear multivariate optimisations [24]. The PSO was used to iteratively modify the properties of the finite element model until it predicted a dispersion curve set that closely matched the set determined from the LV measurements. The PSO algorithm is summarised in the flow chart shown in Figure 6. The 4 material properties used to define the CFRP plate are treated as a position vector $(\vec{x})$ for a particle in a 4 dimensional space (Equation 2)

$$
\vec{x}=\left[\begin{array}{c}
E_{\text {in-plane }} \\
E_{\text {out-of-plane }} \\
G \\
v
\end{array}\right]
$$

Equation 2

At the beginning, each particle is initialised with a random position close to the initial position. Each particle and corresponding 4D position vector is used to evaluate the objective function (Equation 2) which is critical to the optimisation. The objective function essentially compares the finite element model prediction of the dispersion curves at position vector $(\vec{x})$ against the LV obtained dispersion curves. The objective function used here is the $2 \mathrm{D}$ correlation coefficient between the measured and predicted magnitude spectra obtained by 2D FFT of the out-of-plane displacements along the $0^{\circ}$ line shown in Figure 1 and is given in Equations 3 and 4. 
where $r$ is the correlation coefficient given by

$$
r(X, Y)=\frac{\sum_{m} \sum_{n}\left(X_{m n}-\bar{X}\right)\left(Y_{m n}-\bar{Y}\right)}{\sqrt{\left(\sum_{m} \sum_{n}\left(X_{m n}-\bar{X}\right)^{2}\right)\left(\sum_{m} \sum_{n}\left(Y_{m n}-\bar{Y}\right)^{2}\right)}}
$$

Equation 4

here, $X$ and $Y$ are the fields/images to be compared, and the overbar denotes the mean.

The correlation coefficient for the A and S modes are calculated separately and then summed with equal weighting to form the objective function.

After storing the position of the swarm's best $(s b)$ objective function and particles personal best $(x b)$ objective function, the particle velocities can be updated for the next iteration. The calculation of each particle's velocity is central to the performance of the PSO and is given in [21] and is reiterated here in Equation 5.

$$
v=\omega v+\varphi_{p} r_{p}(\boldsymbol{x} \boldsymbol{b}-\overrightarrow{\boldsymbol{x}})+\varphi_{g} r_{g}(\boldsymbol{s} \boldsymbol{b}-\overrightarrow{\boldsymbol{x}}) \quad \text { Equation } 5
$$

where $\omega, \varphi_{p}$ and $\varphi_{g}$ are user selected constants. $r_{p}$ and $r_{g}$ are randomly generated numbers between 0 and 1. The inertial constant $\omega$ has the effect of localising the search when decreased. $\varphi_{p}$ and $\varphi_{g}$ govern the influence from the particle's best position and the swarm's best position respectively. These constants are often modified depending on the behaviour of the objective function; for example, where there are many local minima in the objective function, the inertial constant $\omega$ may be increased to expand the search domain.

Table 2 lists parameters used to configure the PSO. Swarm size and iterations were set conservatively due to the relatively high computational expense of a transient numerical simulation. The inertial constant $\omega$ was set low to allow the PSO to work with a conservative number of iterations. The influence constants $\left(\varphi_{p} \& \varphi_{g}\right)$ were set according to recommendations by Kennedy et al [25].

Table 2 PSO settings.

\begin{tabular}{ll|l}
\multicolumn{2}{c|}{ Swarm size } & 10 \\
\multicolumn{1}{c|}{ Iterations } & 10 \\
$\omega$ & & 0.5 \\
$\varphi_{p}$ & & 1.8 \\
$\varphi_{g}$ & & 1.8
\end{tabular}

The process of updating particle velocities (Equation 5) and evaluating the objective function (Equation 3) is iterated until the target value for the objective function is met. During the iterations, the particle positions can be visualised behaving in a swarm-like behaviour approaching the position of the minimum objective function. 


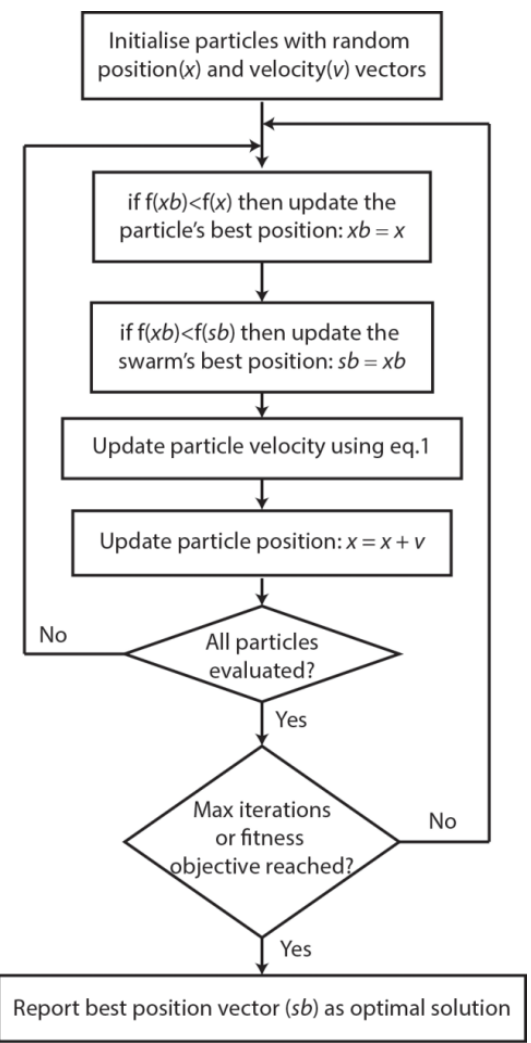

Figure 6 Flow chart describing the PSO algorithm.

\section{Comparison of dispersion curves with new properties}

The initial material properties obtained from published sources and listed in Table 1, were used to calculate the dispersion curves in DISPERSE. Dispersion curves were also calculated by applying a 2D FFT to the displacement-time history acquired experimentally. Figure 7 shows the comparison between the two sets of curves. The need for data subtraction described previously becomes apparent in Figure 7 where the $A_{1}$ and $S_{1}$ modes are seen to overlap in the $300-450 \mathrm{kHz}$ band. The residual $S_{0}$ and $\mathrm{A}_{0}$ modes present in Figures $7 \mathrm{a}$ and $7 \mathrm{~b}$, respectively, can be attributed to experimental error due to misalignment of the scans on the top and bottom surface of the specimen. Significant discrepancies between the numerically and experimentally derived dispersion curves are evident with the latter producing higher wavenumber values in general. In a separate comparison the group velocity of the fundamental modes was measured experimentally and found to be consistently lower than that predicted by DISPERSE, suggesting that the elastic moduli used in the simulation was too high.
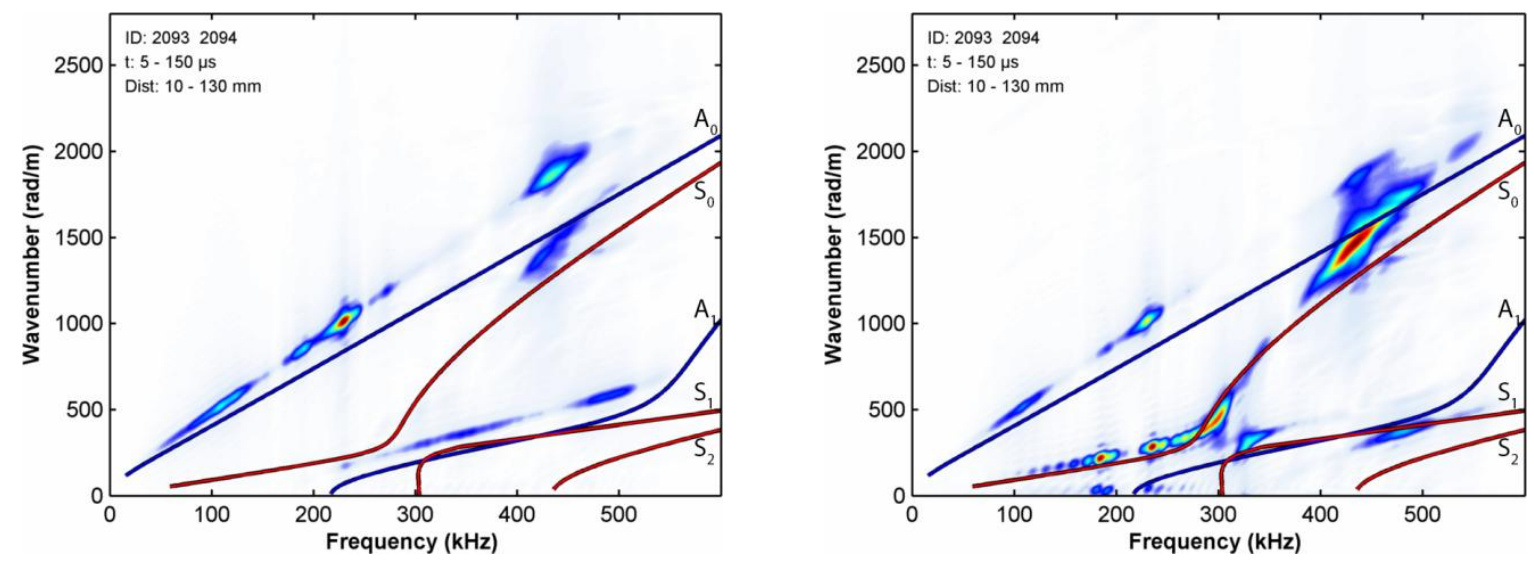
Figure 7 2D FFT: Experimental Lamb modes with DISPERSE curves from initial properties. a) A modes. b) S modes.

Using the published material properties in Table 1 as an initial estimate, the PSO was used to perform a 4 variable optimisation of the material properties of the CFRP plate. The process was run for 10 iterations despite converging much earlier as shown in Figure 8. Figure 8 shows the objective function using the swarm's best position after each iteration. The optimisation is shown to quickly minimise the objective function after 3 iterations. Minor gains were made until the $7^{\text {th }}$ iteration after which no more gains were found and the optimisation was stopped at the $10^{\text {th }}$ iteration resulting in the material properties presented in Table 3. These 10 iterations required 100 COMSOL simulations which took a total of 17 hours of computation on a desktop computer. Substantial improvements in the speed of the process could be made if a Global Matrix Method (GMM) solver were used in place of the COMSOL model; however this option was not available for the present study.

New dispersion curves were calculated in DISPERSE using the optimised material properties in Table 3 and these were overlaid on the experimentally derived dispersion curves. As shown in Figure 9, all modes match closely confirming the validity of the optimised properties. The residual modes resulting from misalignment in the LV data acquisition did not affect convergence of the optimisation algorithm.

The optimised results in Table 3 are substantially different to the initial estimates with the shear and in plane moduli lower by approximately $33 \%$ and 52\% respectively. A discrepancy in the shear modulus was somewhat anticipated as the initial estimate was drawn from generic woven CFRP values taken from the literature, as described previously. The discrepancy in the in-plane modulus is more significant in that the initial estimate was calculated from the manufacturer's properties. The lower inplane stiffness derived from the optimisation procedure is consistent with the waviness of the tows in the weave structure increasing the compliance of the laminate at low loads, as suggested by Malcolm et al [20]. The strain amplitudes in Lamb waves produced by surface bonded piezoelectric elements are typically less than 10 microstrain and often much less, which is consistent with this low load structural response regime.

The wavelengths of the modes considered in this study range from 3-25 mm, which encompasses the RUC length of $7.6 \mathrm{~mm}$ as defined in Figure 3. This raises a question about whether wavelength might have been a factor in the discrepancies shown in Table 3. Also, according to results presented by Poe et al [16], a significant scatter of the elastic modulus of woven composites can arise when the gauge length of the measurement device approaches the length-scale of the RUC. If wavelength were a significant factor in the present study one would expect to have observed a variation in the level of discrepancy as a function of frequency. There does appear to be some evidence of this in Figure 8 with a slightly higher discrepancy at higher frequency for the first order modes, however the effect is modest. 


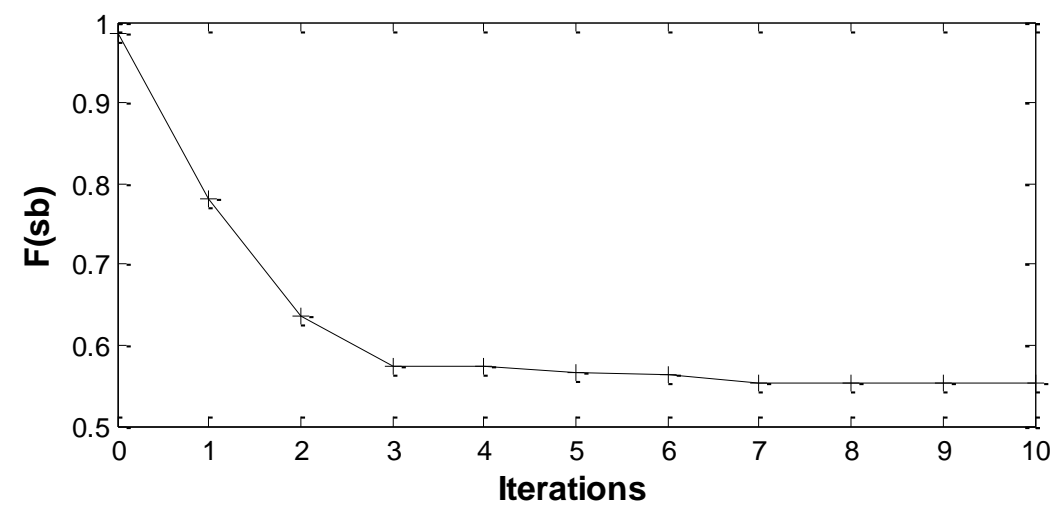

Figure 8 Objective function calculated using the swarm's best (sb) vector at various iteration levels.

Table 3 Material properties before and after optimisation.

\begin{tabular}{l|c|c|c} 
& Initial & Optimised & $\begin{array}{c}\text { Discrepancy } \\
(\%)\end{array}$ \\
$\mathrm{E}_{11}=\mathrm{E}_{33}(\mathrm{GPa})$ & 67 & 44.17 & 51.7 \\
$\mathrm{E}_{22}(\mathrm{GPa})$ & 8.6 & 9.97 & 15.9 \\
$\mathrm{G}_{12}=\mathrm{G}_{13}=\mathrm{G}_{23}(\mathrm{GPa})$ & 5 & 3.33 & 33.3 \\
$v_{12}=v_{13}$ & 0.35 & 0.35 & 0 \\
$v_{23}$ & 0.045 & 0.078 & 73.3
\end{tabular}

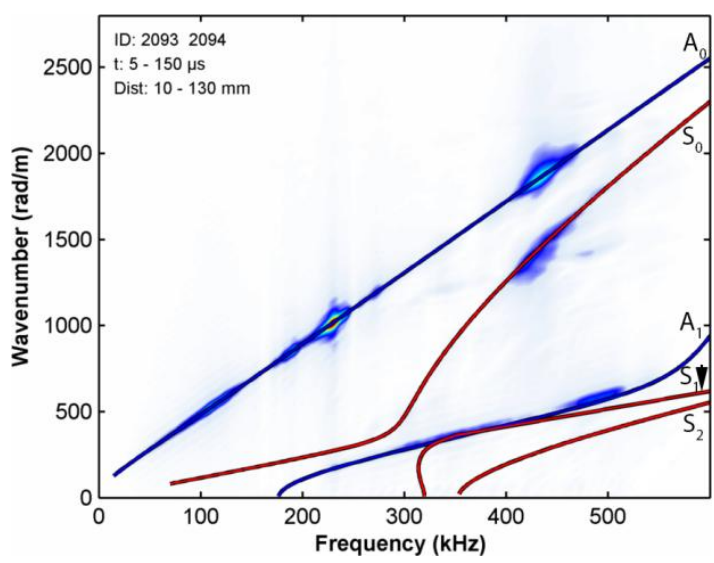

a)

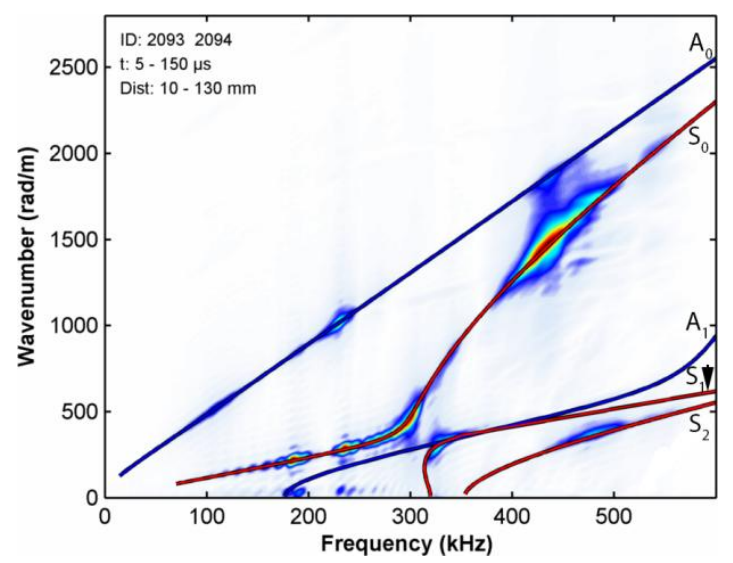

b)

Figure 9 2D FFT: Experimental Lamb modes with DISPERSE curves from optimised properties. a) A modes. b) $S$ modes.

The robustness of the stochastic PSO algorithm was tested by performing a series of optimisations where the initial material properties were perturbed from the published values. The input and output from these additional runs is summarised in Table 4(a) and 4(b).

The results in Table 4(a) summarise the second run and shows that the Young's modulus and shear modulus converged on the same values as the first optimisation (see Table 3) with a maximum discrepancy of $1.8 \%$. However, Poisson's ratio differs by up to $15.1 \%$. The reason for this is the Lamb wave dispersion curves are not very sensitive to Poisson's ratio and will tolerate a large discrepancy. This is demonstrated in Figure 10 where the dispersion curve calculated from properties in Table 4 still results in an excellent match with the experimental data. The only difference is the S1 mode in the vicinity of $325 \mathrm{kHz}$ where better agreement is obtained from the data tabulated in Table 3 (see also Figure 9). However, for all other Lamb wave modes, the set of material properties in Table 3 and 
4 will produce satisfactory models of Lamb wave propagation since they are relatively insensitive to Poisson's ratio.

A third optimisation run was performed with another set of initial values. The results are summarised in Table 4(b). A small change in the value of shear modulus was observed. Figure 11 shows the dispersion curve plotted with these material properties when compared against the experimental data. Figure 11 shows the comparison of the dispersion curves calculated with the elastic properties when compared with the experimental data. As a result of the higher shear modulus, the predicted $\mathrm{A}_{0}$ dispersion curve sits slightly above the experimentally measured one.

Table 4(a) Material properties from second optimisation run with different initial material properties.

\begin{tabular}{l|c|c|c} 
& Initial & Optimised & $\begin{array}{c}\text { Discrepancy from first run } \\
\text { shown in Table 3 }(\%)\end{array}$ \\
$\mathrm{E}_{11}=\mathrm{E}_{33}(\mathrm{GPa})$ & 70 & 44.55 & 0.9 \\
$\mathrm{E}_{22}(\mathrm{GPa})$ & 9 & 10.15 & 1.8 \\
$\mathrm{G}_{12}=\mathrm{G}_{13}=\mathrm{G}_{23}(\mathrm{GPa})$ & 6 & 3.38 & 1.5 \\
$v_{12}=v_{13}$ & 0.35 & 0.297 & 15.1 \\
$v_{23}$ & 0.045 & 0.068 & 12.8
\end{tabular}

Table 4(b) Material properties from third optimisation run.

\begin{tabular}{|c|c|c|c|}
\hline & Initial & Optimised & $\begin{array}{c}\text { Discrepancy from first run } \\
\text { shown in Table } 3(\%)\end{array}$ \\
\hline $\mathrm{E}_{11}=\mathrm{E}_{33}(\mathrm{GPa})$ & 65 & 44.18 & 0.02 \\
\hline $\mathrm{E}_{22}(\mathrm{GPa})$ & 7 & 9.96 & 0.1 \\
\hline $\mathrm{G}_{12}=\mathrm{G}_{13}=\mathrm{G}_{23}(\mathrm{GPa})$ & 4 & 3.20 & 3.9 \\
\hline$v_{12}=v_{13}$ & 0.35 & 0.293 & 16.3 \\
\hline$v_{23}$ & 0.041 & 0.066 & 15.4 \\
\hline
\end{tabular}

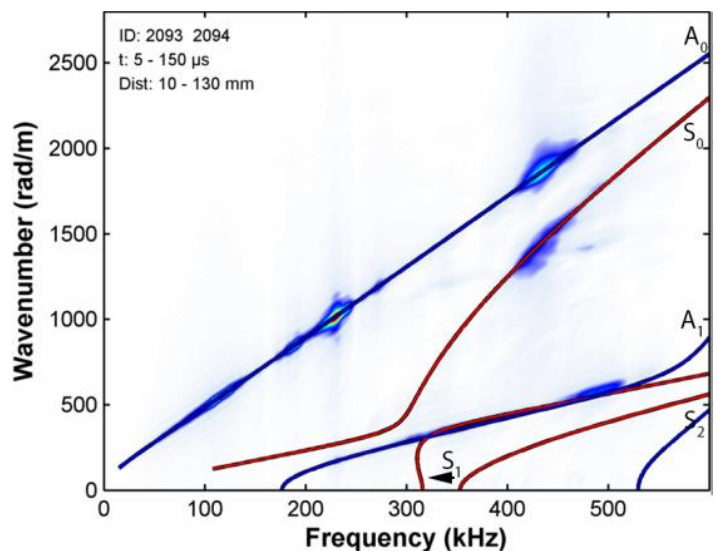

a)

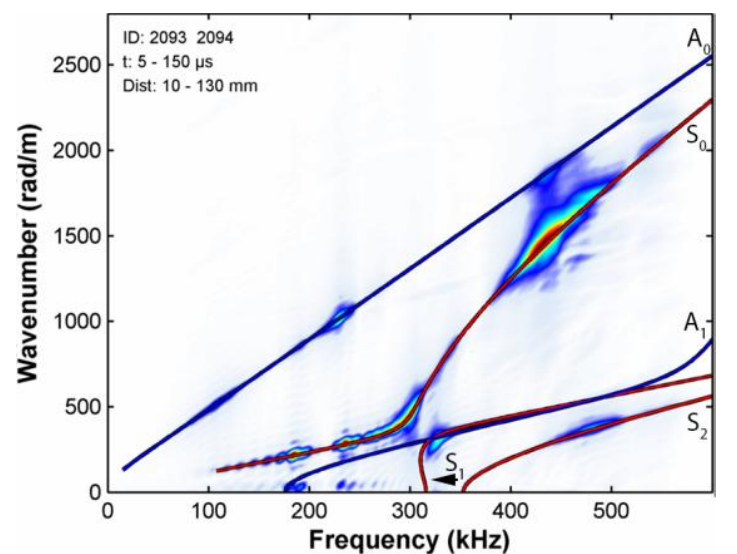

b)

Figure 10 2D FFT: Experimental Lamb modes with DISPERSE curves from optimised properties in Table 4(a). a) A modes. b) $S$ modes. 


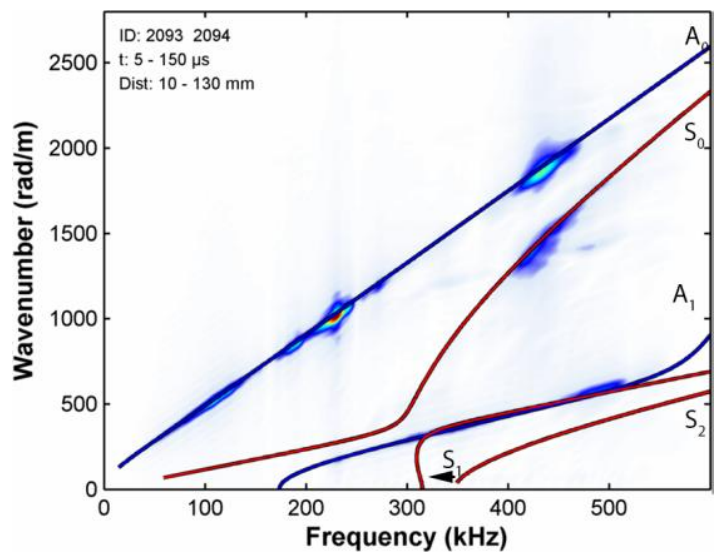

a)

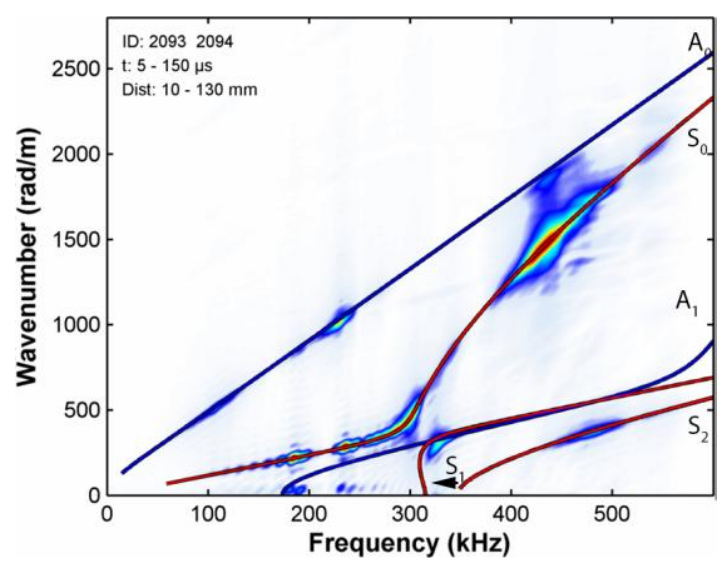

b)

Figure 11 2D FFT: Experimental Lamb modes with DISPERSE curves from optimised properties tabulated in Table 4(b). a) A modes. b) $S$ modes.

\subsection{Quasi-isotropic verification}

As the laminate is nominally quasi-isotropic, a measurement along any arbitrary radial line should in theory be sufficient to determine the material properties. On this basis the optimisation was applied to one set of data only, acquired along the $0^{\circ}$ laminate direction shown in Figure 1 . To test for possible directional variations in panel stiffness, LV scans were also performed along the remaining four directions shown in Figure 1 and the data spectrally decomposed into corresponding sets of dispersion curves. Figure 12 compares these curves to DISPERSE predictions derived from the material properties optimised from the $0^{\circ}$ direction measurements. While the relative amplitudes of the modes vary as a function of direction the dispersion characteristic for each mode is largely consistent across the five directions, confirming the quasi-isotropic structure of the laminate.

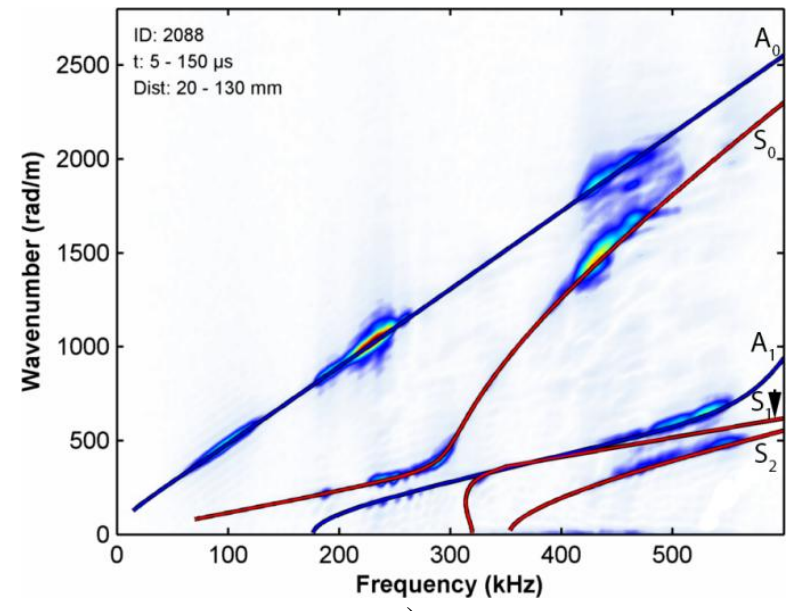

a)

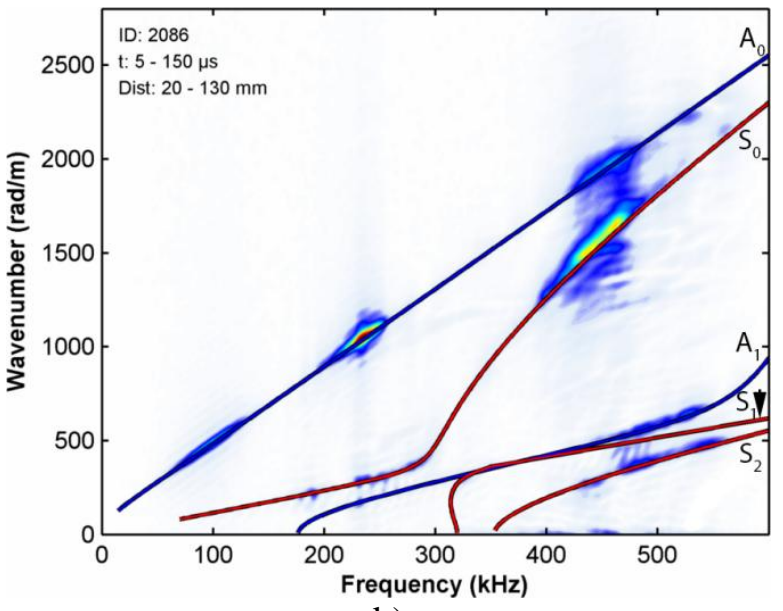

b) 


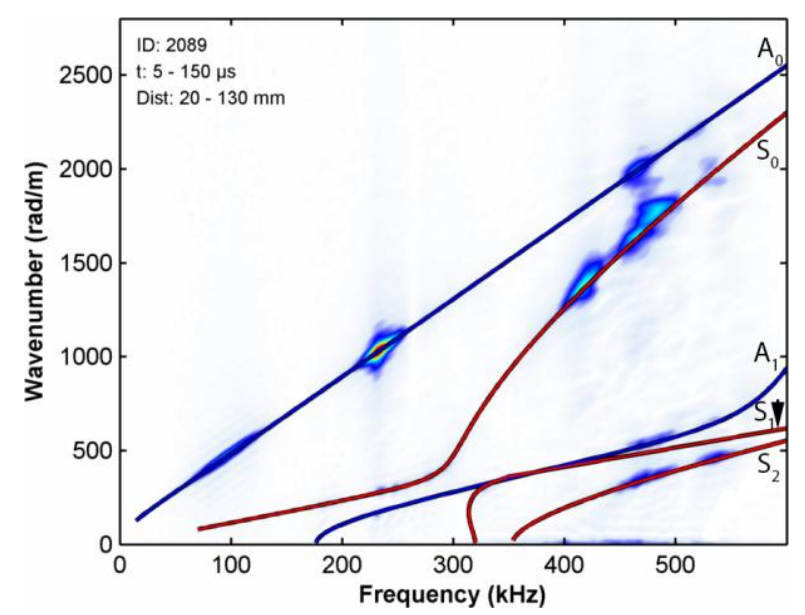

c)

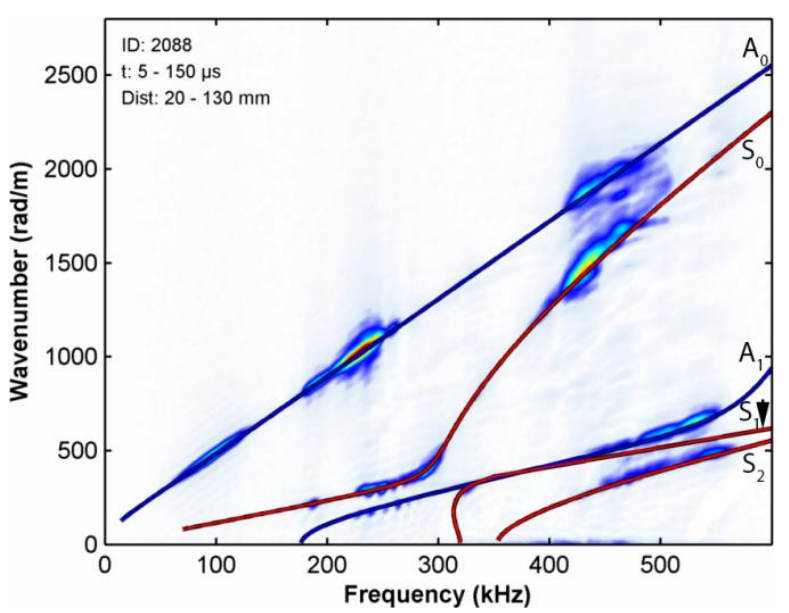

d)

Figure 12 Modal content along various lines indicated in Figure 1. a) $22.5^{\circ}$ b) $45^{\circ}$ c) $67.5^{\circ}$ d) $90^{\circ}$

\section{Conclusions}

In a previous study the use of elastic properties originating from quasi-static testing was unable to accurately model propagating Lamb waves in a woven composite [15]. In response, it was proposed that a material property set different from that obtained by quasi-static testing was needed to model propagating Lamb waves. In order to determine elastic properties in the Lamb wave propagation regime, a procedure was proposed based on optimisation to determine the elastic properties of woven composite laminates. The method was based on measured dispersion characteristics of fundamental and first order symmetric and antisymmetric Lamb wave modes in the frequency-thickness product range 0.2-2 MHz-mm. It was found that the elastic properties required to model Lamb wave propagation were different by as much as $52 \%$ from those supplied by the manufacturer. The discrepancy was attributed to the fibre waviness in woven composites producing a load dependent stiffness that is at a minimum under small loads. The findings affirm that a reliance on published properties or standard quasi-static methods of measurement is inadequate for accurate computational simulation of Lamb waves in woven composite materials.

\section{Acknowledgements}

The authors gratefully acknowledge the Office of Naval Research for the support of this work under the Naval International Cooperative Opportunities in Science and Technology Program (Grant No. N62909-12-1-7005).

\section{References}

1. Ong, W.H. and W.K. Chiu, Enhancement of Lamb wave-based in situ structural health monitoring through guided local geometry changes. Structural Health Monitoring, 2013. 12(4): p. 339-358.

2. Ong, W.H. and W.K. Chiu, Numerical modelling of scattered Lamb waves through varied damage size in challenging geometry. Structural Health Monitoring, 2013. 12(3): p. 278-295. 
3. Ostachowicz, W., P. Kudela, and M. Radzienski, Experimental Validation of Algorithms for Wave Propagation Modeling in 2D and 3D Structures Based on the Spectral Element Method. Structural Health Monitoring 2011: Condition-Based Maintenance and Intelligent Structures, Vol 1 and Vol 2, 2011: p. 915-922.

4. Packo, P., T. Bielak, A.B. Spencer, W.J. Staszewski, T. Uhl, and K. Worden, Lamb wave propagation modelling and simulation using parallel processing architecture and graphical cards. Smart Materials and Structures, 2012. 21(7).

5. Staszewski, W.J. and B.C. Lee, Modelling of Lamb waves for damage detection in metallic structures: Part II. Wave interactions with damage. Smart Materials \& Structures, 2003. 12(5): p. 815-824.

6. Zhang, X., S.F. Yuan, and T. Hao, Lamb wave propagation modeling for structural health monitoring. Frontiers of Mechanical Engineering in China, 2009. 4(3).

7. Ong W H, R.C., Rajic N, Chiu W K. Determination of elastic properties in a plate by Lamb wave analysis and particle swarm optimisation. in 7th International conference on materials for advanced technologies. 2013. Suntec Singapore

8. Lowe, M., DISPERSE, 1996-2007, Imperial College, Department of Mechanical Engineering: London.

9. Ng, C.T. and M. Veidt, Scattering characteristics of Lamb waves from debondings at structural features in composite laminates. Journal of the Acoustical Society of America, 2012. 132(1): p. 115-123.

10. Li, F., H. Peng, X. Sun, J. Wang, and G. Meng, Wave Propagation Analysis in Composite Laminates Containing a Delamination Using a Three-Dimensional Spectral Element Method. Mathematical Problems in Engineering, 2012. 2012: p. 19.

11. Naik, R.A., Analysis of woven and braided fabric reinforced composites, 1994, NASA: Hampton, Virginia. CR-194930

12. Dixit, A., H.S. Mali, and R.K. Misra, Unit cell model of woven fabric textile composite for multiscale analysis. International Tribology Conference Malaysia 2013, 2013. 68: p. 352-358.

13. Gommers, B., I. Verpoest, and P. VanHoutte, Modelling the elastic properties of knittedfabric-reinforced composites. Composites Science and Technology, 1996. 56(6): p. 685-694.

14. Chou, T.W., Microstructural design of fiber composites. Micromechanics of Advanced Materials, 1995: p. 413-419. 
15. Ong, W.H., W.K. Chiu, N. Rajic, and C. Rosalie, The inadequacy of elastic properties from tensile tests for Lamb wave analysis, in The 6th Internatioal Conference on Computational Methods (ICCM2015)2015: Auckland, New Zealand.

16. Poe, C.C., H.B. Dexter, and I.S. Raju, A review of the NASA textile composites research, 1997, NASA Langley Technical Report Sever.

17. Chen, B. and T.W. Chou, Local elastodynamic stresses in the unit cell of a woven fabric composite. Archive of Applied Mechanics, 2000. 70(6): p. 423-442.

18. Green, S.D., M.Y. Matveev, A.C. Long, D. Ivanov, and S.R. Hallett, Mechanical modelling of $3 D$ woven composites considering realistic unit cell geometry. Composite Structures, 2014. 118: p. 284-293.

19. Mishra, R., Meso-scale finite element modeling of triaxial woven fabrics for composite inplane reinforcement properties. Textile Research Journal, 2013. 83(17): p. 1836-1845.

20. Malcom, A.J., M.T. Aronson, and H.N. Wadley, Three-dimensionally woven glass fiber composite struts: characterization and mechanical response in tension and compression. Journal of Composite Materials, 2015.

21. Shi, Y.H. and R. Eberhart, A modified particle swarm optimizer. 1998 Ieee International Conference on Evolutionary Computation - Proceedings, 1998: p. 69-73.

22. Hexcel, HexPly M18/1 Product Data, 2007, Hexcel.

23. Alleyne, D.N. and P. Cawley, A 2-Dimensional Fourier-Transform Method for the Quantitative Measurement of Lamb Modes. Ieee 1990 Ultrasonics Symposium : Proceedings, Vols 1-3, 1990: p. 1143-1146.

24. Berg, F.V.D., An Analysis of Particle Swarm Optimizers, in Faculty of Natural and Agricultural Science2001, University of Pretoria: Pretoria.

25. Kennedy, J. and R. Eberhart, Particle swarm optimization. 1995 Ieee International Conference on Neural Networks Proceedings, Vols 1-6, 1995: p. 1942-1948. 